\title{
Research Paper \\ Relationship Between Organizational Agility, Innovative Work Behaviors, and Job Satisfaction Among the Second-grade High School Teachers
}

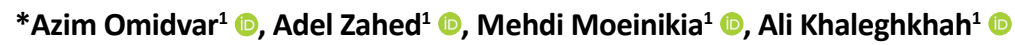

1. Department of Educational Scineces, Faculty of Eductional Sciences and Psychology, University of Mohaghegh Ardebili, Ardebil, Iran.

\begin{tabular}{|c|c|}
\hline $\begin{array}{l}\text { Use your device to scan } \\
\text { and read the article online }\end{array}$ & Citation: Omidvar, A., Zahed, A., Moeinikia, N., \& Khaleghkhah, A. (2021). [Relationship Between Organizational Agility, \\
\hline 口ith & Innovative Work Behaviors, and Job Satisfaction Among the Second-grade High School Teachers (Persian)]. Journal of School \\
\hline thin & Psychologh and Institutions, 9(4), 86-95. https://doi.org/10.32598/JSPI.9.4.7 \\
\hline 口ifing & dol'https://doi.org/10.32598/JSPI.9.4.7 \\
\hline
\end{tabular}

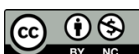

Article Info:

Received: 07 May 2019

Accepted: 14 Nov 2019

Available Online: 01 Jan 2021

Key words:

Job satisfaction, Organizational agility, Innovative work behaviors

\section{ABSTRACT}

Objective The present study explored the relationship between organizational agility, innovative work behaviors, and job satisfaction in second-grade secondary school teachers in Ardebil City, Iran.

Methods This was a descriptive correlational study. The statistical population of this research was all secondary school teachers in Ardebil City, Iran, in the academic year of 2016-2017. Among them, 129 teachers were selected by cluster random sampling method. The research tools were the Organizational Agility Scale (Zhang et al., 2000), the Innovative Work Behavior Questionnaire (Holman et al., 2011), and the Job Satisfaction Scale (Berifield et al., 1951). Data analysis was performed using Pearson's correlation coefficient and regression analysis method.

Results The obtained results indicated a significant positive correlation between organizational agility, innovative work behaviors, and teachers' job satisfaction. Furthermore, the results of regression analysis revealed that roughly $0.42 \%$ of the total variance of job satisfaction was predicted based on organizational agility and innovative work behaviors $(P<0.001)$.

Conclusion The obtained results suggested that organizational agility and innovative work behaviors were related to the prevention of teachers' job satisfaction, which calls for the attention of authorities and educators to these variables.

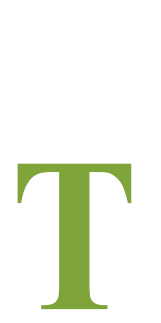

\section{Extended Abstract}

\section{Introduction}

eachers are the focus and important training factors in educational organizations and directly target the goal of the organization. Teachers' behavior determines whether schools do their job well, also, the quality and development of education are strongly dependent on the effectiveness of teachers (Amani \&
Shabahang, 2017). Besides, any organization that seeks to increase its productivity and efficiency particularly values the job satisfaction of its employees, as job satisfaction plays an essential role in enhancing the organization's performance (Mudor \& Tooksoon, 2011). The low job satisfaction of employees reliably indicates the deteriorating situation of an organization (Vernica, 2011). Therefore, teachers' job satisfaction is crucial for the success of teachers, students, community, and school systems. Saiti and Papadopoulos (2015) defined job satisfaction as a person's positive expression of a job position.

\section{* Corresponding Author:}

\section{Azim Omidvar, PhD. Student}

Address: Department of Educational Scineces, Faculty of Eductional Sciences and Psychology, University of Mohaghegh Ardebili, Ardebil, Iran. Tel: +98 (45) 33519981

E-mail:azim_omidvar@yahoo.com 
Teachers encounter potential negative incidents, such as student behavior, teaching stress, high workload, and the regulations of the school board and government. Work environment problems affect physical, emotional, and social health and cause the conditions of depression, restlessness, and physical problems (Hatami, 2017).

Moreover, organizational agility results from being immensely conscious of changes in both internal and external environments. Organizational agility can take an effective form if is accompanied by the ability to use resources to respond to the changes at a convenient and flexible time and also the organization be able to implement the changes (Braunscheidel \& Suresh, 2009). Teachers who are among the most important specialists for the future of society will increase the level of self-esteem and conflict with work by increasing job satisfaction against stressful life events and occupational environments. This would be possible by creating "innovation" in their job, developing new ideas, and nurturing and applying them (Azimpour \& Jalilian, 2016). Innovative behaviors, as behaviors that bring about change themselves, are related to job satisfaction. These behaviors represent the creation of new or different things and are defined by the orientation for change because they are associated with the creation of a new product, service, thought, and process. This study aimed to survey the relationship between organizational agility, innovative work behaviors, and job satisfaction among the second-grade high school teachers of Ardabil City.

\section{Materials and Methods}

This was a descriptive-correlational research. Also, the statistical population included all the second-grade high school teachers of Ardabil City in the academic year of 2017-2018. From this population, 129 teachers were randomly selected using the cluster sampling method. The study measures included the organizational agility questionnaire (Shafifi \& Zhang, 1999), innovative work behavior questionnaire (Hoffman, Bynum, Piccolo, \& Sutton 2011), and job satisfaction questionnaire (Brayfield \& Rothe, 1951).

\section{Results}

Demographic findings showed that 87 teachers were male and 42 were female. Of these participants, 35, 41, and 53 had under 10 years, 10 to 20 years, and 20 to 30 years of work experience, respectively. Besides, 81, 35, and 13 teachers were permanent, temporary, and contract employees, respectively. Moreover, the education levels of 55, 36, and 38 teachers were bachelor's degrees, master's degrees, and doctoral candidacy, respectively.
Descriptive findings indicated the Mean $\pm \mathrm{SD}$ of $64.26 \pm 10.40,56.12 \pm 17.20$, and $33.26 \pm 18.09$ for the total scores of job satisfaction, organizational agility, and innovative work behavior, respectively. Besides, the inferential findings showed that the teachers' job satisfaction was significantly associated with organizational agility $(\mathrm{r}=0.54, \mathrm{P}$ $<0.01)$ and innovative work behaviors ( $\mathrm{r}=-0.60, \mathrm{P}<0.01)$. The variables of organizational agility and innovative work behavior predicted approximately $42 \%$ of the variance of teachers' job satisfaction. Also, the F-ratio confirmed the significance of the regression equation of teachers' job satisfaction based on the variables of organizational agility and innovative work behavior. Organizational agility and innovative work behavior variables positively and significantly predicted teachers' job satisfaction with the beta values of $0.212(\mathrm{P}<0.01, \mathrm{t}=2.132)$ and $0.231(\mathrm{P}<0.01, \mathrm{t}=2.324)$, respectively. The obtained betas showed that in terms of predictive power, the variables of innovative work behavior and organizational agility are the first and second predictors, respectively.

\section{Discussion and Conclusion}

This study indicated that the increase of organizational agility and innovative work behaviors helps to promote job satisfaction in teachers. These results call for the attention of the education authorities and officials towards such variables. Understanding and anticipating changes in the business environment, agile organizations benefit from the opportunities resulting from their restructuring and reorganization. Setting the stage for long-term and sustainable success in organizations cause an optimum and healthy atmosphere in the organization, and all of these factors lead to job satisfaction among teachers. Overall, the present study showed that organizational agility and innovative work behaviors are variables related to teachers' job satisfaction.

The limitation of the study to a specific geographical area (Ardabil City) and the use of self-report scales to collect data may expose the results to social utility bias. Therefore, it is suggested that research be conducted in other geographical areas to generalize the results. To further generalize the results and achieve cause-and-effect relationships, future studies are recommended to conduct such research on the teachers of other educational levels, and if possible, investigate the relations experimentally. Also, the research suggestions of this study include increasing the mental resilience of teachers in the workplace, motivating teachers to work with better quality using the methods of success, and increasing hope among teachers about their career's future. 


\section{Ethical Considerations}

\section{Compliance with ethical guidelines}

All ethical principles are considered in this article. The participants were informed about the purpose of the research and its implementation stages. They were also assured about the confidentiality of their information and were free to leave the study whenever they wished, and if desired, the research results would be available to them.

\section{Funding}

The paper was extracted from a research project of the University of Mohaghegh Ardabili.

\section{Authors' contributions}

All authors equally contributed to preparing this article.

\section{Conflicts of interest}

The authors declared no conflict of interest. 
رابطه جِابكى سازمانى و رفتارهاى كارى نوآورانه با رضايت شعلى معلمان مقطع متوسطه يايه دوم شمبر إربيل

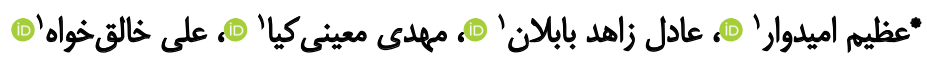
ا.كروه آموزش علوم تربيتى، دانشكده علوم تربيتى وروانشناسى، دانشكاه محقق اردبيلى، اردبيل، ايران.

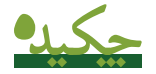

هدف يُروهش حاضر با هدف بررسى رابطه جابكى سازمانى و رفتارهاى كارى نوآورانه با رضايت شغلى معلمان مقطع متوسطه يايه دوم شهر اردبيل انجام كرفتي.

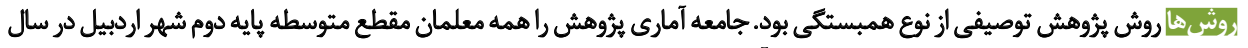

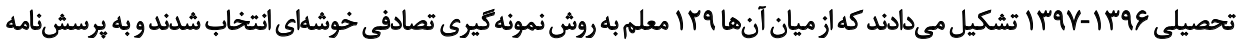

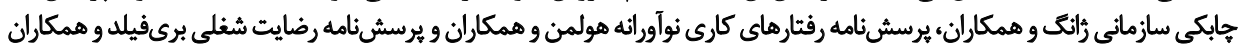

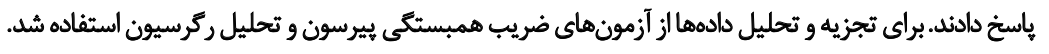

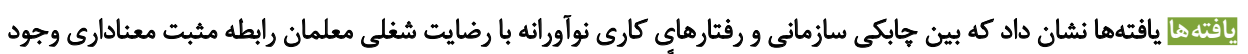

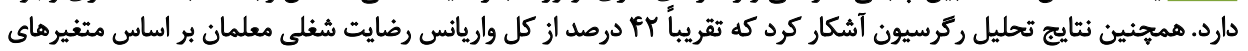

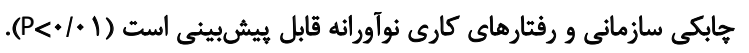

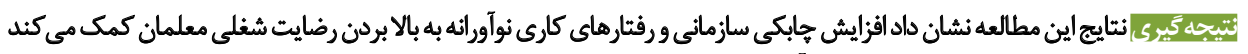

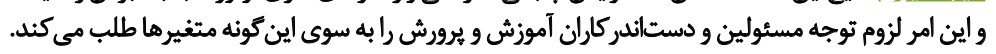

اطلاعات مقاله:

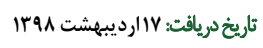
تاريخ يذيرش: بr آبان

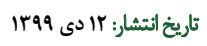

كليبوأوها: رضايث شغلى، جابكيى

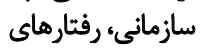
كارى نوآورانه

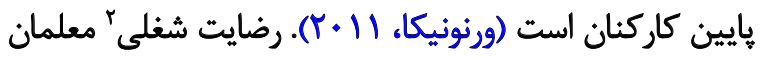

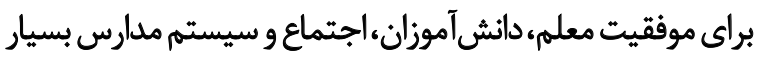

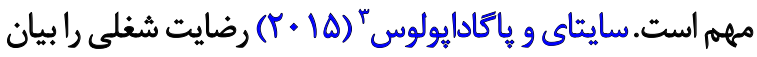

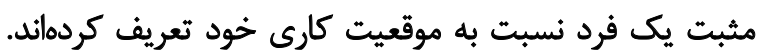

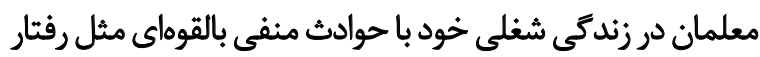

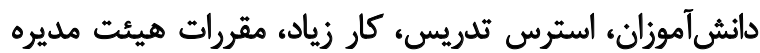

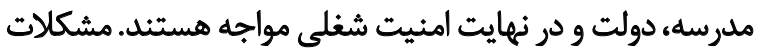

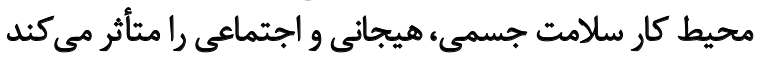

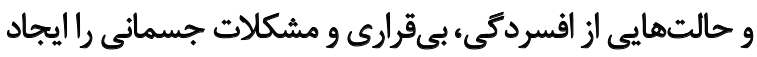

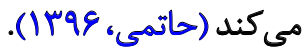

رضايت شعلى از مهمترين موضوعاتى است كه در هر سازمان

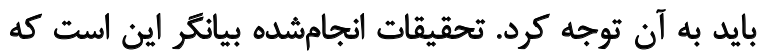

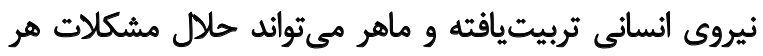

2. Job satisfaction

3. Saiti \& Papadopoulos

1. Mudor \& Tooksoon

مقدمه

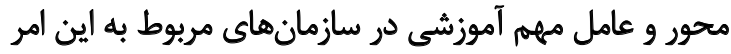

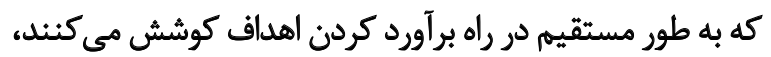

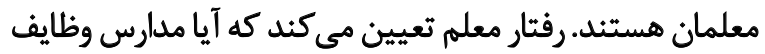

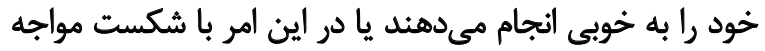

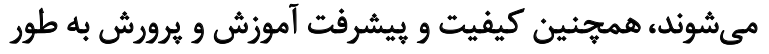

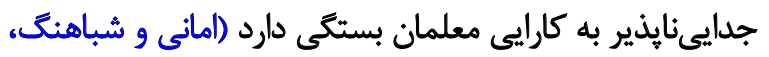

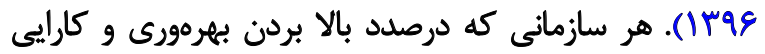

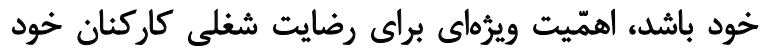

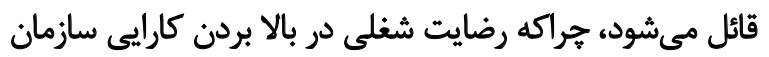

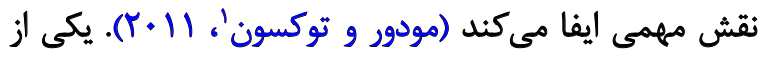
مطمئنترين نشانههاي تباهى اوضاع يك سازمان، رضايت شغلى تيلى 


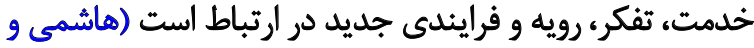

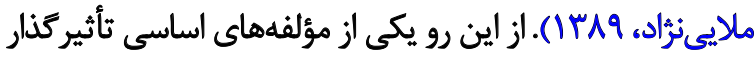

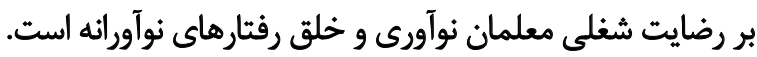

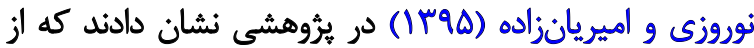

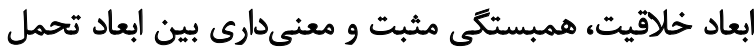

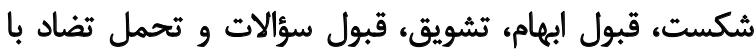

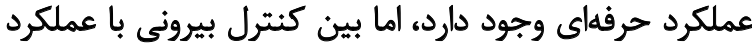

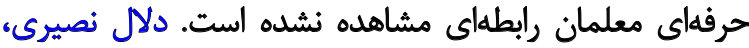

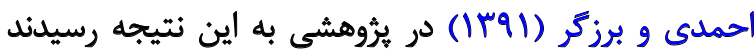

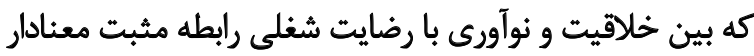

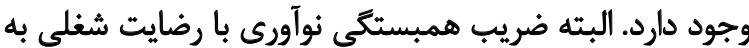

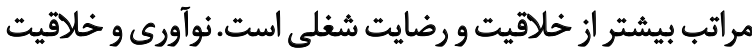

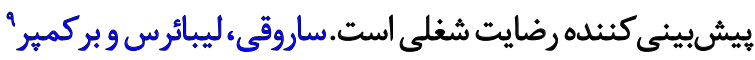

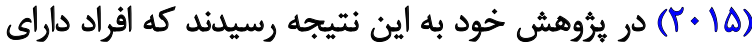

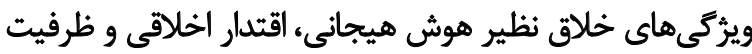

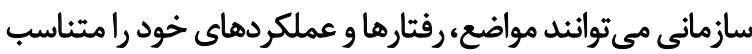

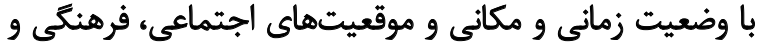

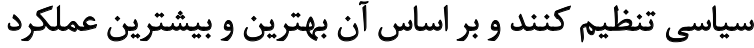

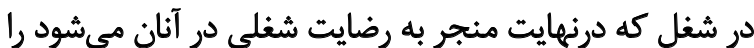

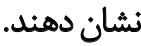

شغل معلمى به دليل ترتيب نسل آينده و اثراتى كه مى توانيد

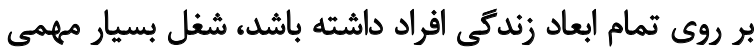

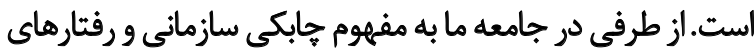

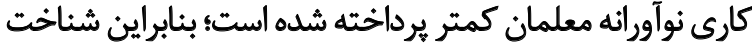

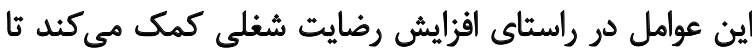

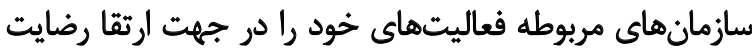

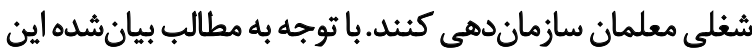

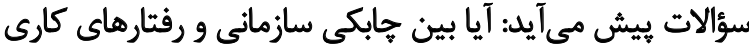

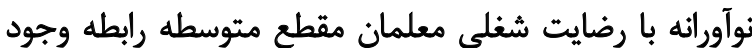

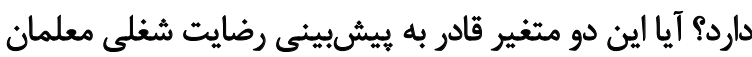

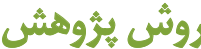

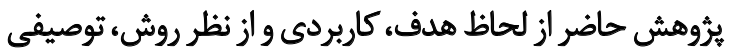

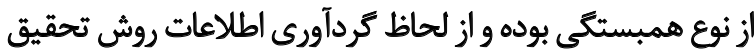

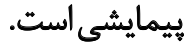

جامعه، نمونه و روش نمونه كيرى: جامعه آمارى يثروهش حاضر

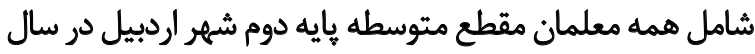

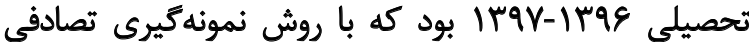

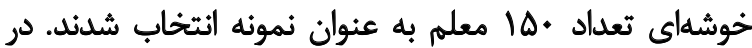

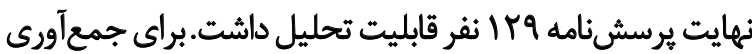

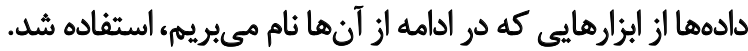

سازمان باشد؛ بنابراين هر فرد بايد شغلى را انتخاب كند كه علاوه

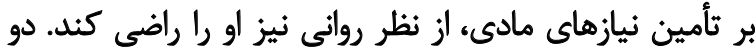

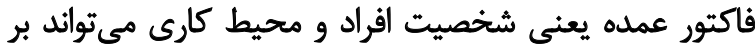

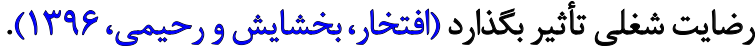
در اين ميان يكى از عواملى كه نقش مههمى در رضايت شغلى

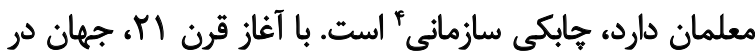

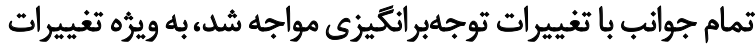

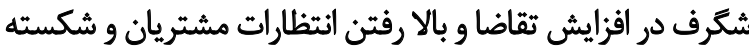

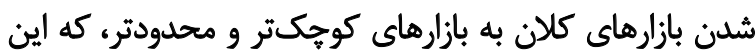

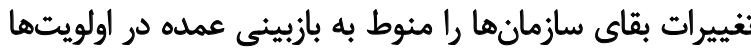

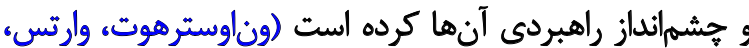

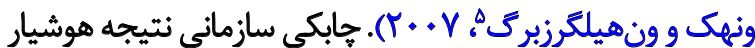

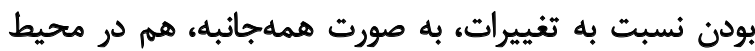

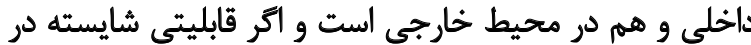

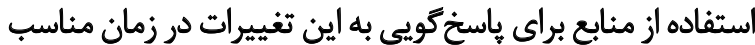

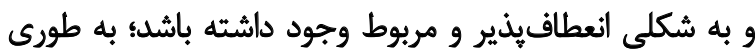

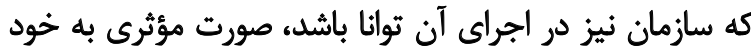

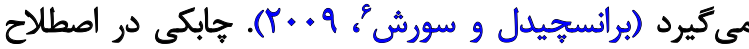

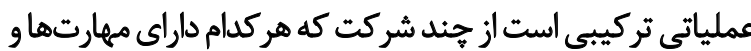

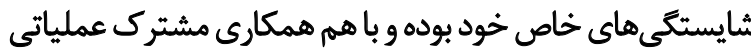

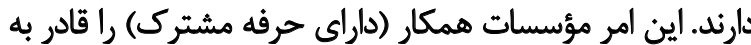

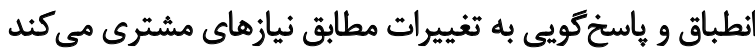

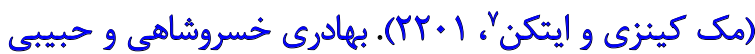

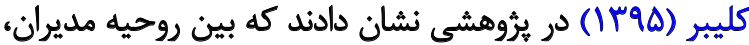

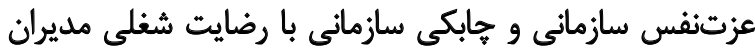

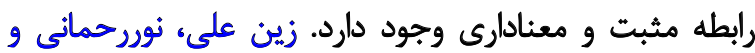

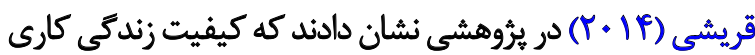
اثر توجهبرانكيزى در رضايت شغلى معلمان دارد.

مي توان انتظار داشت معلمان كه از مهمثرين متخصصان براى

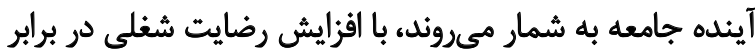

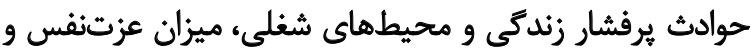

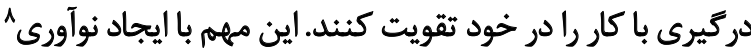

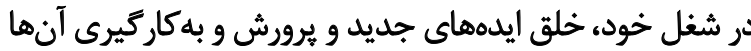

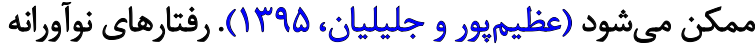

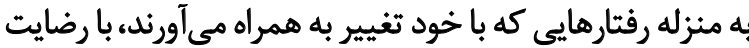

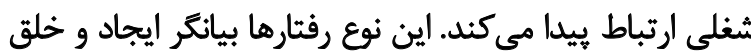

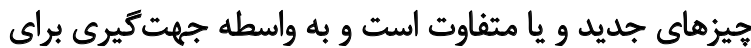

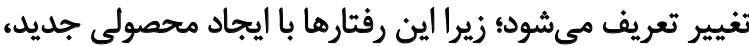
4. Organizational agility
5. Van Oosterhout, Waarts, Van Heck, \& Van Hillegersberg
6. Braunscheidel \& Suresh
7. McKenzie \& Aitken
8. Innovative 
جدول 1. آمارههاي توصيفى رضايت شغلى

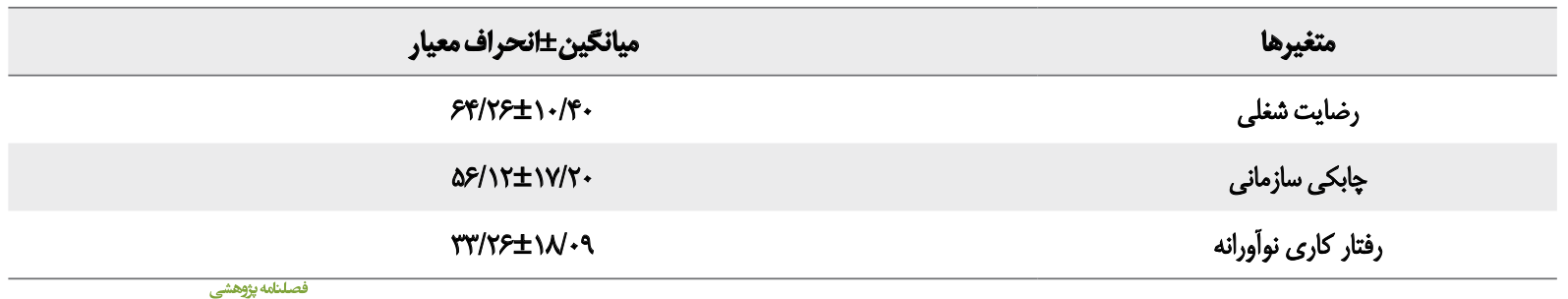

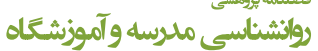

ضريب آلفاي كرونباخ برابر 191• به دست آمده است. دادههاي

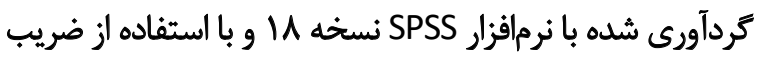
همبستى ييرسون و تحليل ركرسيون به شيوه كامبه كام تجزيه

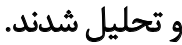

C. los

در يُروهش حاضر

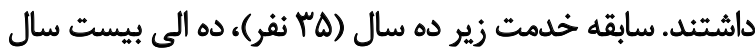

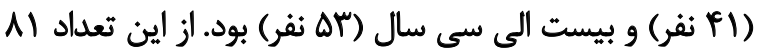

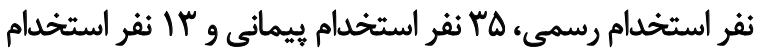
قراردادى بودند. سطح تحصيلات ها نفر نفر آنها ليسانس، عب نفر نفر

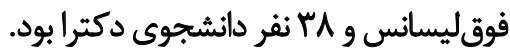

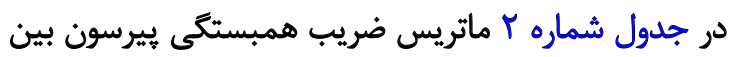

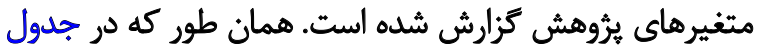

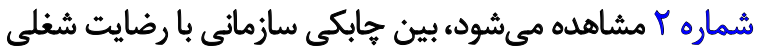

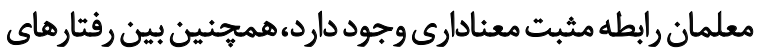

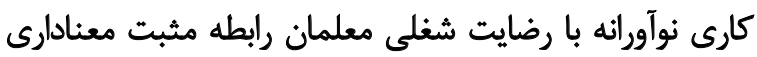

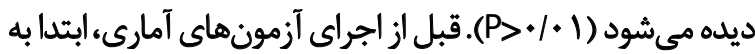

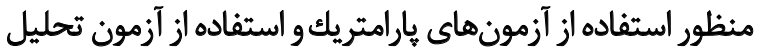

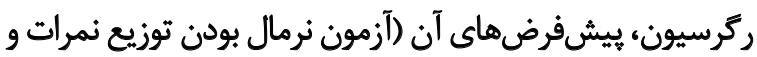

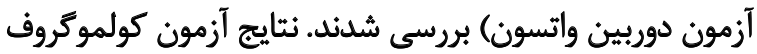

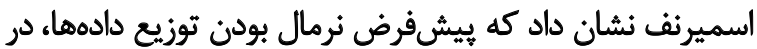

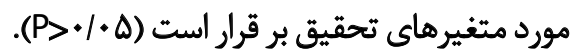

همان طور كه در جدول شماره ب مشاهده مي مشود، نسبت

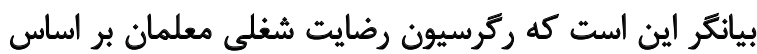

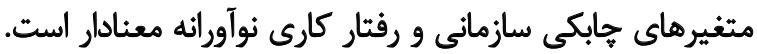

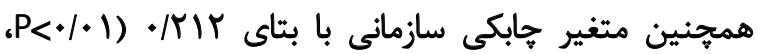

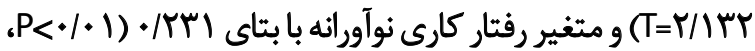

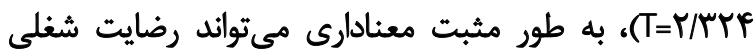

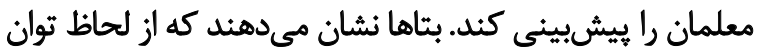

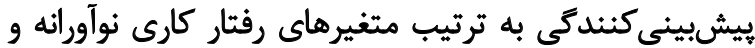
جابكى سازمانى قرار دارند.
يرسشنامه رضايت شغلى ": براي اندازهيرى رضايت شغلى

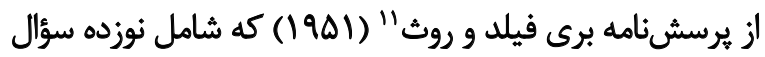

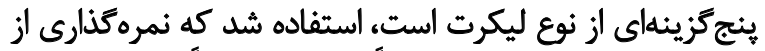

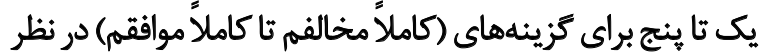

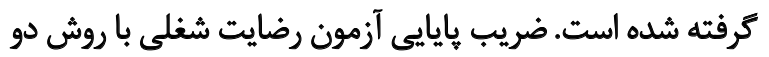

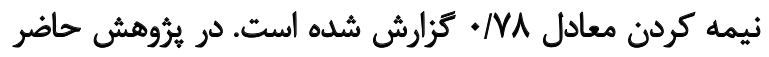

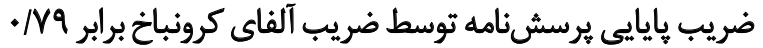
به دست آمده است.

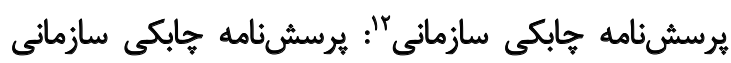

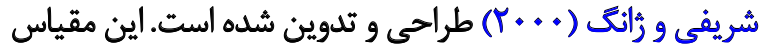

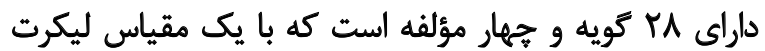

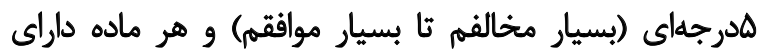

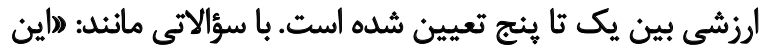

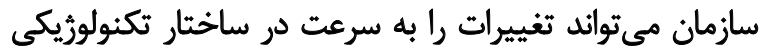

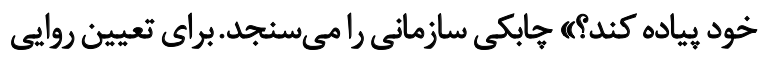

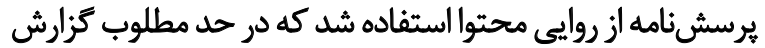

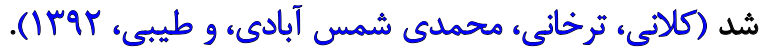

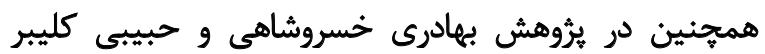

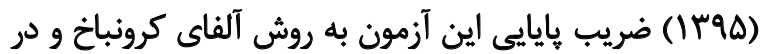

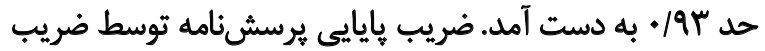

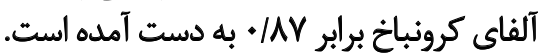

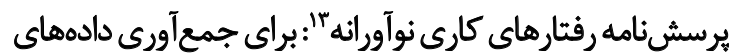

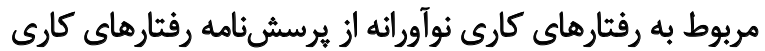

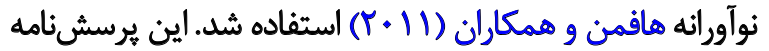

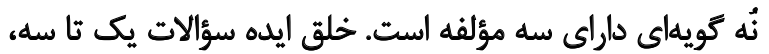

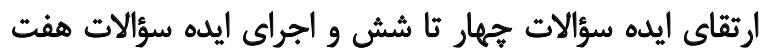

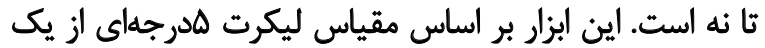

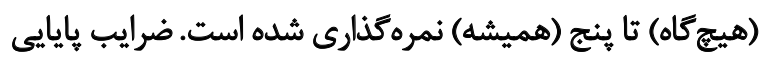

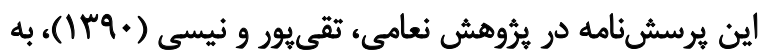

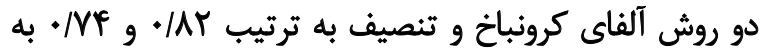

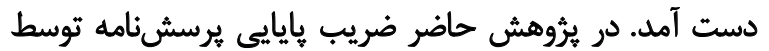

10. Job Satisfaction Questionnaire

11. Brayfield \& Rothe

12. Organizational Agility Questionnaire

13. Innovative Work Behaviors Questionnaire 
جدول r. ضرايب همبستكى بين هابكى سازمانى با رضايت شغلى معلمان

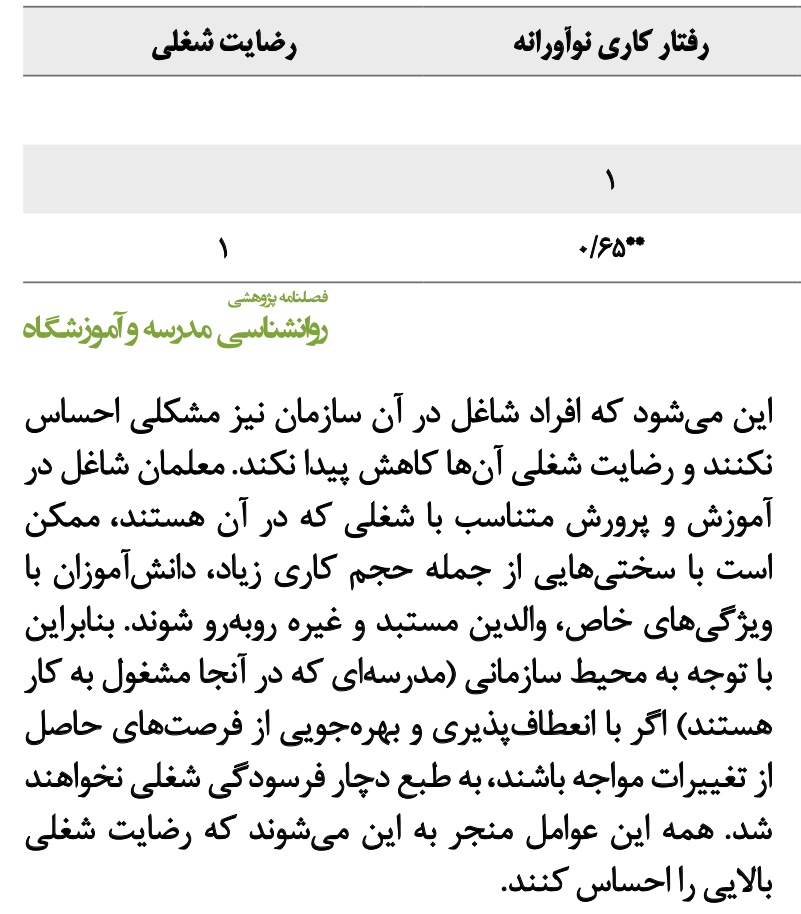

يافته دوم يثروهش نشان داد كه بين رفتارهاى كارى نوآورانه

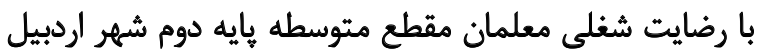

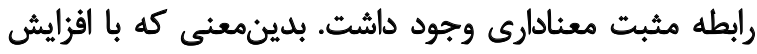

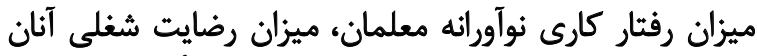

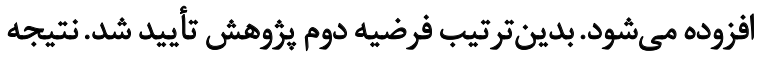

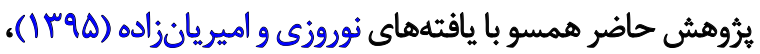

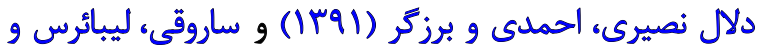

$$
\text { بركمير (10 - (Y) است. }
$$

در تبيين يافته حاضر مى توان اين كونه استدلال كرد كه زمانى

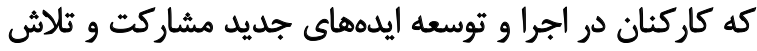

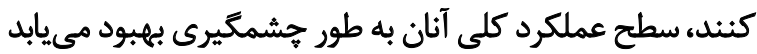

\begin{tabular}{|c|c|}
\hline هابكى سازمانى & متغيرها \\
\hline 1 & هابكى سازمائى \\
\hline$\cdot / \Delta \Delta$ & رفتار كارى نوآورانه \\
\hline \multirow[t]{2}{*}{.$/\left.\Delta\right|^{p e n}$} & رضايت شغلى \\
\hline & \\
\hline
\end{tabular}

رئروهش حاضر با هدف سنجش رابطه هابكى سازمانى و وانو

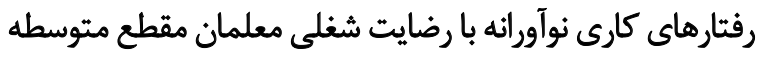

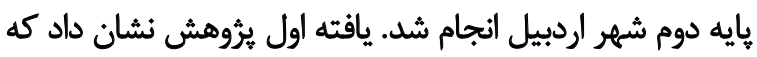

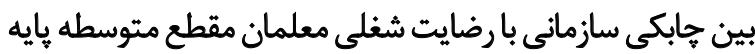

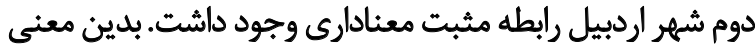

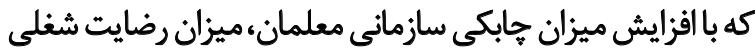

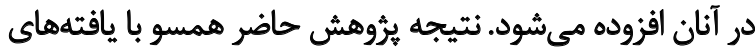

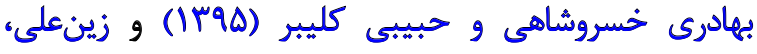

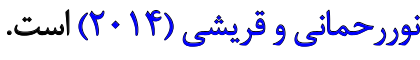

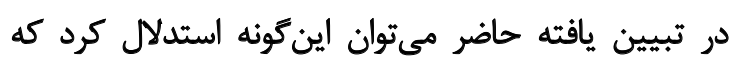

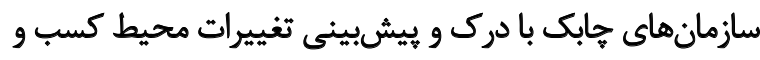

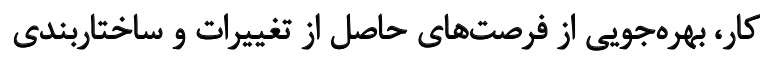

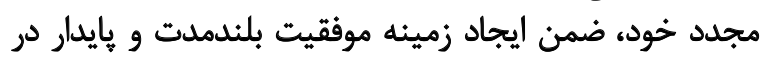

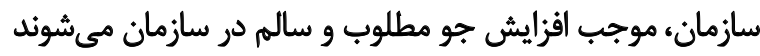

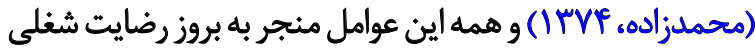

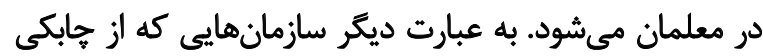

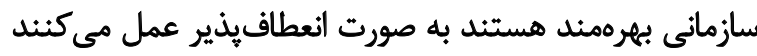

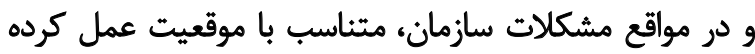
و شيوههاى مقابلهاي كارآمد اتخاذ مي كنيند. اين عمل منجر باته

جدول r. تحليل واريانس مدل ركرسيون جهت بيشبينى رضايت شغلى معلمان

\begin{tabular}{|c|c|c|c|c|c|}
\hline Sig. & $\mathbf{F}$ & MS & df & SS & منيع تغييرات \\
\hline $.1 . .1$ & $9 v / .9$ & вqтя/99. & $r$ & $\mid \Psi \wedge V \Psi / q \Lambda$. & ركرسيون \\
\hline- & - & $V \cdot / r \Delta q$ & Tra & $|M| \cdot|/ A| F$ & باقىمانده \\
\hline - & - & - & IrA & roqva/vaY & كل \\
\hline Sig. & $T$ & $\beta$ & STE & B & متغيرهاى ييشبين \\
\hline $.1 .+1$ & $10 / r \cdot \varphi$ & - & a/wa & 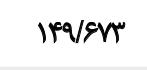 & مقدار ثابت \\
\hline .1 .1 & $r / N T$ & . MIT & 正 &. & هابكى سازمانى \\
\hline . & $r / M r P$ & ו ו & . & . THAP & رفتار كارى نوآورانه \\
\hline
\end{tabular}

ووائشناسى ملارسه و آموزشغاه 


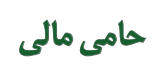

مقاله حاضر بركرفته از طرح يثوهشى درون سازمانى دانشكاه

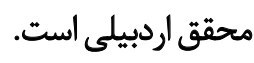

$$
\text { مشاركت نويسندكًان }
$$

تمام نويسندكان در طراحى، اجرا و نتارش همه بخشهاى ئروهش حاضر مشاركت داشتهاند.

$$
\text { تعارض منافع }
$$

بنابر اظهار نويسندكان اين مقاله تعارض منافع ندارد.
و در راستاى هرجه بهتر عملكرد كارى خود، به جستوجوى إنو

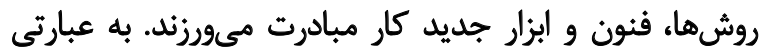

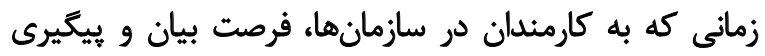

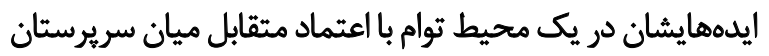

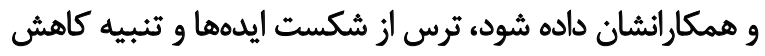

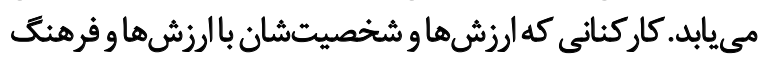

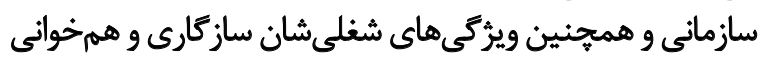

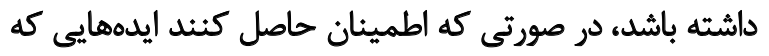

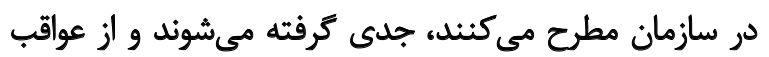

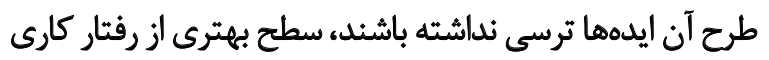

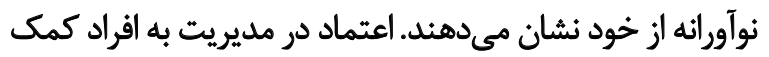

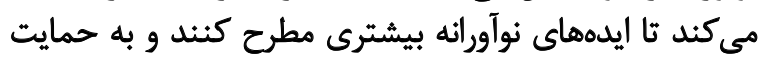

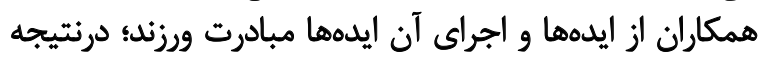

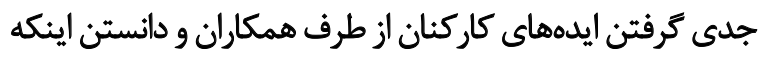

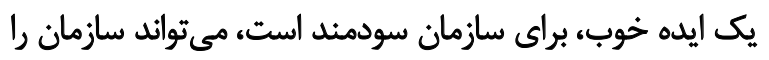

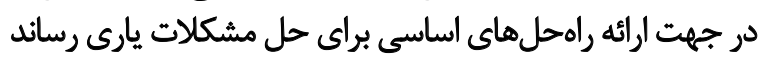

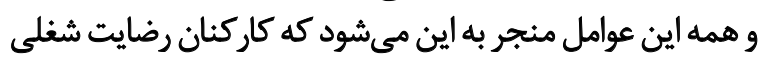

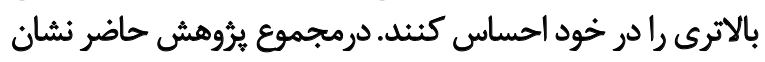

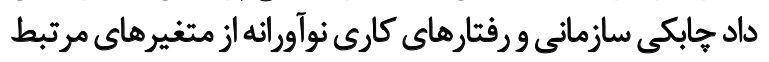
با رضايت شغلى معلمان است.

محدود بودن به منطقه جغرافيايي حاص (شهر اردبيل) و محدود

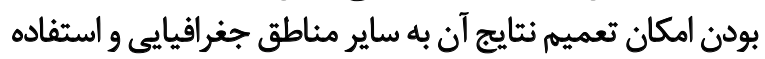

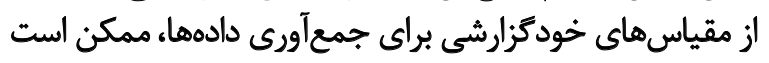

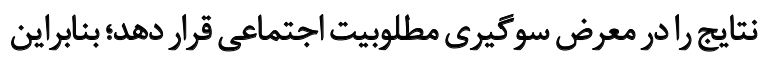

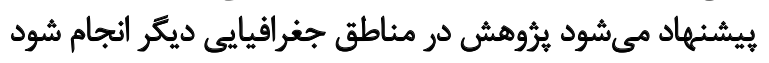

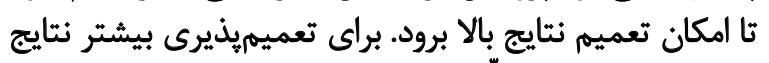

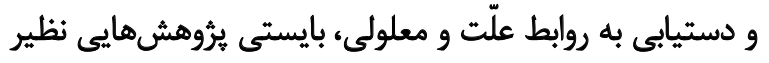

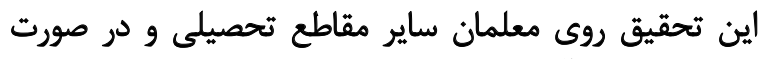

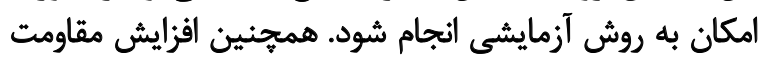

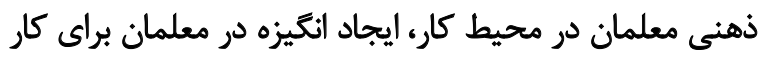

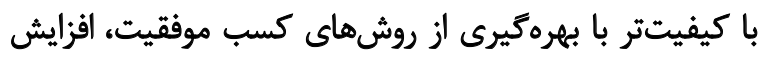

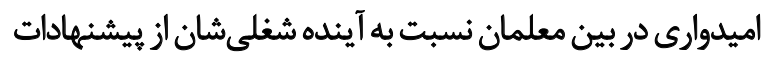
هُؤوهشى اين تحقيق است.

مالاحظاث أخاقي

$$
\text { بيروى ائز اصول اخلاق بثوهش }
$$

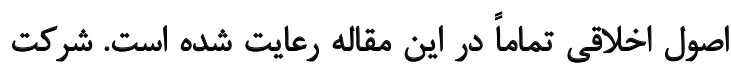

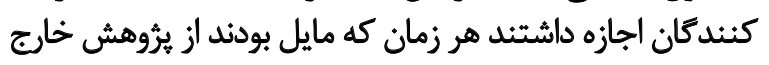

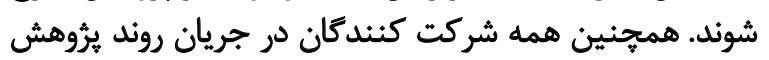

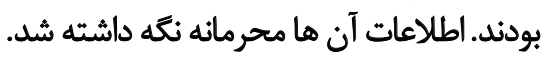




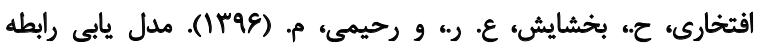

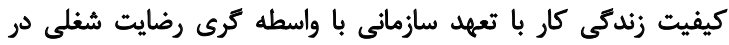

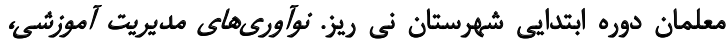

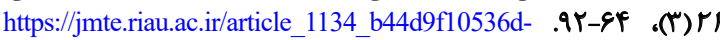
d894573111de34843f28.pdf

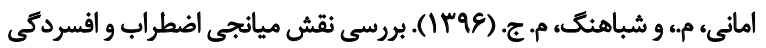

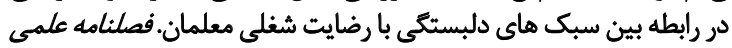
http://tkj.ssu.ac.ir/article-1-767-fa. . تخصصى طب كان

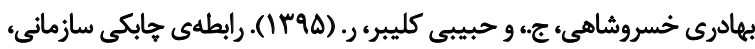

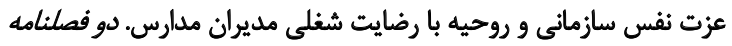

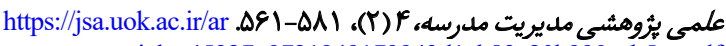
ticle_45327_9721949179943d1eb83c20b990aeb5ce.pdf

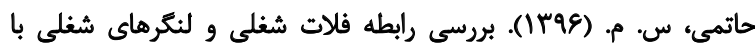

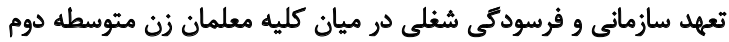

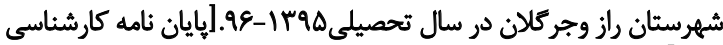
ارشد]. اروميه: دانشعاه ارومئه

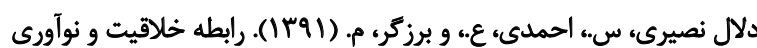

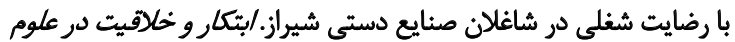

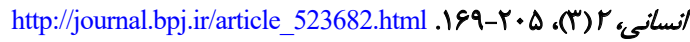

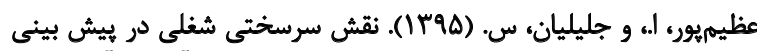

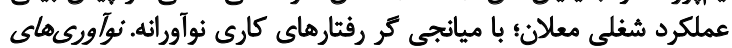

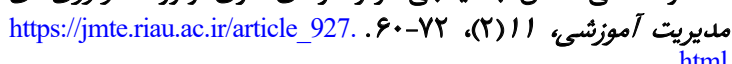

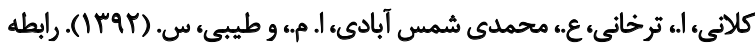

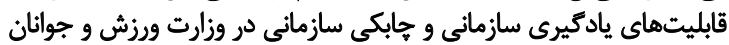

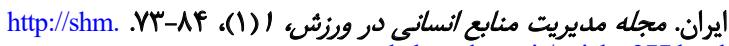
shahroodut.ac.ir/article_277.html

محمدزاده، ع. (ITVF) (مديريث توسعه. جاب اول، تهران: انتشارات سمت.

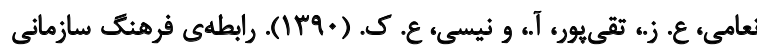

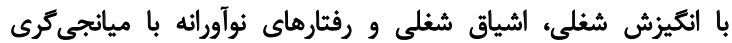

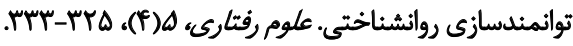

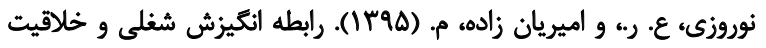

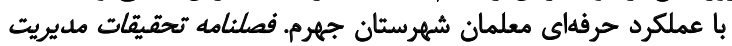

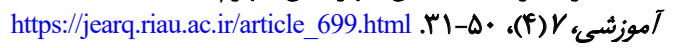

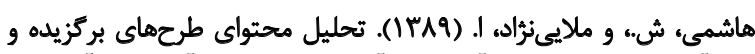

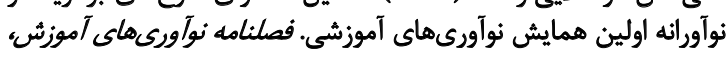
https://www.magiran.com/paper/908685. 


\section{References}

Amani, M., \& Shabahang, M., 2017. [Investigating the role of anxiety and depression mediators in relationship between teachers' attachment styles and job satisfaction (Persian)]. Occupational Medicine Quarterly Journal, 9(2), 59-70. https://www.sid.ir/en/ journal/ViewPaper.aspx?ID $=542557$

Azimpour, E., \& Jalileans, S. (2016). [Role Job Hardiness in predicting job performance, with the mediation of innovative work behaviors Role Job Hardiness in predicting job performance, with the mediation of innovative work behaviors (Persian)]. The Journal of Modern Thoughts in Education, 11(2), 72-60. https://jmte. riau.ac.ir/m/article 927.html?lang=en

Bahadori Khosroshhi, J., \& Habibi, R. (2017). [The relationship between organizational agility, organizational self-esteem, morale and job satisfaction of Prinsipals (Persian)]. Job Safety Analysis, 4(2), 165-85. https://jsa.uok.ac.ir/article_45327_en.html

Braunscheidel, M. J., \& Suresh, N. C. (2009). The organizational antecedents of a firm's supply chain agility for risk mitigation and response. Journal of Operations Management, 27(2), 119-40. [DOI:10.1016/j.jom.2008.09.006]

Brayfield, A. H., \& Rothe, H. F. (1951). An index of job satisfaction. Journal of Applied Psychology, 35(5), 307. [DOI:10.1037/ h0055617]

Dallal, N. S., Ahmadi, E., \& Barzegar, M. (2013). [Study the relationship between creativity and job satisfaction in Shiraz handcraft employees (Persian)]. Journal of Innovation and Creativity in Human Science, 2(3), 169-204. https://www.sid.ir/en/journal/ ViewPaper.aspx?ID=344133

Eftekhari, H., Bakhshayesh, A. R., \& Rahimi, M. (2017). [Modeling of the relationship between the quality of work life and organizational commitment by means of job satisfaction among elementary school teachers of Neyriz (Persian)]. The Journal of Modern Thoughts in Education, 12(3), 29-46. https://jmte.riau.ac.ir/article_1134.html?lang=en

Hashemi, S. H., \& Mollaeenezhad, A. (2011). [Content analysis of the selected and innovative plans presented at the $1^{\text {st }}$ Iranian conference on educational innovations (Persian)]. Quarterly Journal of Educational Innovations, 9(36), 126-49. https://www.sid.ir/en/ journal/ViewPaper.aspx?ID=249290

Hatami, H. (2017). [Investigating the relationship between occupational plots and occupational anchors with organizational commitment and burnout among all Secondary Teachers in the city of Raz Vojgordlan in the academic year of 2011-2012 (Persian)]. [MA. thesis]. Urmia: Urmia University.

Hoffman, B. J., Bynum, B. H., Piccolo, R. F., \& Sutton, A. W. (2011). Person-organization value congruence: How transformational leaders influence work group effectiveness. Academy of Management Journal, 54(4), 779-96. [DOI:10.5465/amj.2011.64870139]

Kalani, A., Tarkhani, E., Mohammadishamsabadi, A., \& Tayebi, S. (2013). [The relationship between organizational learning capabilities and organizational agility in Iran's Ministry of Sports and Youth (Persian)]. Human Resource Management in Sport Journal, 1(1), 75-87. http://shm.shahroodut.ac.ir/article 277.html

McKenzie, J., \& Aitken, P. (2012). Development of digital product catalogue for enabling agility in a manufacturing organization. Strategic Human Resources Review, 11(6), 12-31. [DOI:10.1108/14754391211264794]
Ali Mohamadzadeh, K., Darabi, A., \& Jafari, M. (2017). The relationship between organizational agility and organizational health in hospitals affiliated to Shahid Beheshti University of Medical Sciences. Journal of Payavard Salamat, 11(3), 276-86. http:// payavard.tums.ac.ir/article-1-6281-en.html

Mohammadzadeh, A. (1994). [Development Management (Persian)]. Tehran: Samt Publication.

Mudor, H., \& Tooksoon, P. (2011). Conceptual framework on the relationship between human resource management practices, job satisfaction and Turnover. Journal of Economics and Behavior Studies, 2(2):41-9. [DOI:10.22610/jebs.v2i2.220]

Naemi, A. A., Nisi, A., Taghipour, A. [The relationship between organizational culture and job motivation, job motivation and innovative behaviors mediated by psychological empowerment (Persian)]. Paper presented at Conference on Management and Leadership Challenges in Iranian Organizations. Iran, Esfahan; 15 July 2019. https:// civilica.com/doc/91441/

Norozi, A., \& Amirianzadeh, M. (2016). [The relationship between job motivation and creativity with professional performance of teachers in Jahrom city (Persian)]. Educational Management Research, 7(4), 31-50. https://jearq.riau.ac.ir/article_699.html

Saiti, A., Papadopoulos, Y. (2015). School teachers' job satisfaction and personal characteristics. International Journal of Educational Management, 29(1), 73-97. [DOI:10.1108/IJEM-05-2013-0081]

Sarooghi, H., Libaers, D., \& Burkemper, A. (2015). Examning the relationship between creativity and innovation: A meta-analysis of organizational, cultural, and environmental factors. Journal of Business Venturing, 30(5), 714-31. [DOI:10.1016/j.jbusvent.2014.12.003]

Sohrabian, A., Modaresi, S., \& Yousefi, F. (2018). [The relationship between organizational climate and job satisfaction among occupied nurses in psychiatric hospitals of Qods, Tohid and Besat (Persian)] Shenakht Journal of Psychology and Psychiatry, 4(4), 34-43. https:// www.sid.ir/en/journal/ViewPaper.aspx?ID=568494

Van Oosterhout, M., Waarts, E., Van Heck, E., \& Van Hillegersberg, J Business Agility: Need, Readiness and Alignment with IT Strategies. In: Desouza KC, editor. Agile information systems: Conceptualization, Construction, and management. Elsevier Butterworth-Heinemann.

Veronica, D. (2011). Stress and job satisfaction among university, International conference of scientific paper AFASES. Germany, Brasov; 2011 May 26-28.

Zeinali Pour, H., Noor Rahmani, M., \& Qureshi, A. (2014). The study of relationship between quality of work life and job satisfaction of high school Teachers in Bandar Abbas. American Journal of Life Science. 2(1), 118-24. http://citeseerx.ist.psu.edu/viewdoc/download?doi=10 1.1.1081.370\&rep $=$ rep1\&type $=$ pdf

Sharifi, H., \& Zhang, Z. (1999). A methodology for achieving agility in manufacturing organisations: An introduction. International Journal of Production Economics, 62(1-2), 7-22. [DOI:10.1016/S09255273(98)00217-5] 